This is the final peer-reviewed accepted manuscript of:

M. Crescentini et al., "Experimental Assessment of a Broadband Current Sensor based on the X-Hall Architecture," 2020 IEEE International Instrumentation and Measurement Technology Conference (I2MTC), Dubrovnik, Croatia, 2020, pp. 1-6, doi: 10.1109/I2MTC43012.2020.9128994. The final published version is available online at DOI:

http://dx.doi.org/10.1109/12MTC43012.2020.9128994

Rights / License:

The terms and conditions for the reuse of this version of the manuscript are specified in the publishing policy. For all terms of use and more information see the publisher's website.

This item was downloaded from IRIS Università di Bologna (https://cris.unibo.it/)

When citing, please refer to the published version. 


\section{Experimental Assessment of a Broadband Current Sensor based on the X-Hall Architecture}

\author{
Marco Crescentini \\ DEI, Cesena Campus \\ University of Bologna \\ Cesena, Italy \\ m.crescentini@unibo.it
}

\author{
Roberto Canegallo \\ Analog \& Smart Power TR\&D \\ STMicroelectronics \\ Castelletto, Italy \\ roberto.canegallo@unibo.it
}

\author{
Roberta Ramilli \\ ARCES, Cesena Campus \\ University of Bologna \\ Cesena, Italy \\ roberta.ramilli2@unibo.it
}

\author{
Aldo Romani \\ DEI, ARCES, Cesena Campus \\ University of Bologna \\ Cesena, Italy \\ aldo.romani@unibo.it
}

\author{
Gian Piero Gibiino \\ DEI \\ University of Bologna \\ Bologna, Italy \\ gianpiero.gibiino@unibo.it \\ Marco Tartagni \\ DEI, ARCES, Cesena Campus \\ University of Bologna \\ Cesena, Italy \\ marco.tartagni@unibo.it
}

\author{
Marco Marchesi \\ Analog \& Smart Power TR\&D \\ STMicroelectronics \\ Castelletto, Italy \\ marco.marchesi@st.com \\ Pier Andrea Traverso \\ $D E I$ \\ University of Bologna \\ Bologna, Italy \\ pierandrea.traverso@unibo.it
}

\begin{abstract}
The X-Hall sensor is presented, characterized and proposed as a viable architecture for silicon-integrated, broadband, current/magnetic-field measurements. The $\mathrm{X}$-Hall architecture overcomes the methodological bandwidth limit of state-of-the-art Hall-effect sensors by replacing the typically used spinning-current technique with a DC bias-based, passive offset compensation technique, which is less effective from an absolute standpoint but presents the key feature of being frequency independent.

Three different prototypes have been realized and experimentally characterized in both static and dynamic operation. Static characterization demonstrates a competitive residual offset of the $\mathrm{X}$-Hall sensor with respect to spun Hall sensors operated at high frequency. Even though physical simulations reveal a theoretical bandwidth of $200 \mathrm{MHz}$ for the $X$-Hall sensor, experimental dynamic characterization on the prototypes identifies the presence of additive dynamic perturbations limiting the sensor bandwidth, which are attributable to the practical implementation of the prototypes. However, it is possible to compensate these perturbations through a vector differential measurement model, so that a bandwidth of $4 \mathrm{MHz}$ is demonstrated, which is the broadest bandwidth ever achieved by a Hall-effect based sensor, to the best knowledge of the authors.
\end{abstract}

Keywords—broadband current measurement, Hall effect, Hall probe, current sensor, magnetometers.

\section{INTRODUCTION}

There is a general emerging need in power electronics to detect and measure fast-varying currents and magnetic fields. This need is driven by the development of innovative power devices, which are able to operate at higher frequencies and higher power rates, and the emerging of very fast response (VFR) applications, such as dynamic voltage scaling in microprocessors [1] and high-frequency AC inverters [2]. In VFR applications, the power converter must be able to track the target output voltage in the microsecond and beyond, thus requiring a fine time resolution in the current measurement.

Standard solutions for broadband current measurement imply the use of AC-coupled coils, which are cumbersome and lose DC information on the current/magnetic field. Integrating the current sensor in the same chip together with the power device would have considerable impacts on the final application, since it allows to reduce the occupied space and the weight of the final system, and it is less sensitive to external interferences ${ }^{1}$. The Hall-effect sensor is a viable technology to achieve integration with the power device, but it is usually limited in bandwidth to hundreds of $\mathrm{kHz}$ or, maximum, $1 \mathrm{MHz}$ [3]-[5].

This paper presents the X-Hall architecture and experimentally investigates its potentialities and weaknesses. The X-Hall architecture aims at widening the acquisition bandwidth of Hall-based current sensors by removing the spinning-current technique and minimizing the capacitive load seen by the Hall probe. Minimization of the capacitive load is facilitated by the removal of all the switches required by the spinning-current technique. At the same time, the XHall sensor lowers the high intrinsic offset of the Hall probe by using passive offset compensation at the probe level, in contrast to [6] where it is implemented at the sensor level. According to [5] and [7], replacing the spinning-current biasing with a purely DC-biasing allows the Hall sensor to theoretically achieve a bandwidth of tens to hundreds of $\mathrm{MHz}$. Concurrently, the X-Hall sensor must demonstrate to be able to reduce the intrinsic offset to an acceptable level.

The X-Hall theoretical idea was presented, for the first time, at the 2018 IMEKO World Congress [8] with a limited set of preliminary experimental data. In this paper, an exhaustive static and dynamic experimental characterization carried out on manufactured prototypes is presented and discussed. Section II describes the theory of the X-Hall architecture [8], detailing the passive offset compensation. Static and dynamic characterizations of the implemented prototype are reported in Section III and Section IV, respectively. Finally, conclusions are drawn in Section V.

\section{The X-HALl ARChitecture}

\section{A. Topological Aspects of the X-Hall Probe}

The X-Hall probe could be realized in standard silicon technology (e.g. BCD8 smart-power technology) by a lowlydoped n-type well surrounded by a grounded $\mathrm{p}$-type well. The use of an $\mathrm{n}$-well as the active sensing region is preferable to a p-well because it presents higher current-related sensitivity $S_{I}$

\footnotetext{
${ }^{1}$ Power systems are usually employed in very noisy environments. For instance, inverters used with motors in electric vehicles suffer from strong electronic interferences, vibrations, thermal stresses, etc.
} 
according to [4] and [5]. The encapsulation of the n-type well in the p-type well is unavoidable since it assures electric isolation from the substrate. An example of the vertical section of the probe is shown in Fig. 1-a.

In the X-Hall architecture, the active region is octagonally shaped and accessible by a total of 8 contacts: 4 large contacts $(B, T, L, R)$ used to bias the probe, and 4 small contacts $(1,2$, $3,4)$ used to sense the Hall voltage. A top view of the octagonal Hall probe is shown in Fig. 1-b. In contrast to spun Hall sensor, the contacts are dedicated to a single purpose (biasing or sensing) so that they can be optimized according to their specific function. The bias contacts are large-sized to minimize the access resistance and are orthogonally oriented to the edges of the probe so as to maximize the sensitivity [9]. The sense contacts are small-sized to minimize the parasitic capacitance associated with the contacts and to maximize the sensitivity [9]. Sense contacts cannot be shrunk too much, otherwise, the intrinsic offset of the probe will be deteriorated [9].

\section{B. Biasing Scheme and Passive Offset Reduction}

In the X-Hall configuration, two equal DC currents $\left(I_{A}=\right.$ $I_{B}$ ) are fed to two opposite bias contacts (e.g. T and B) while the other two bias contacts are connected to ground (e.g. R and L) and all the sense contacts are electrically floating. This biasing scheme (Fig. 1-b) generates a global current density distribution with uniform magnitude along the inscribed circumference, yet exciting four orthogonal directions (Fig. 2). From a general perspective, this biasing scheme concurrently polarizes the sensor in four orthogonal directions, like a "static" current-spinning technique. Actually, the orthogonal bias currents flow in different regions of the probe, thus, it is reasonable to assume that the local inhomogeneities will not be corrected.

According to this biasing scheme, it is also possible to identify two inner Hall-effect probes inside the octagonal active region. One inner probe (probe A) is placed below the horizontal axis of symmetry of the global probe while the other inner probe (probe B) is placed above the same axis of symmetry (see Fig. 1-b). These two probes are biased by the same nominal current value and share the bias contacts $\mathrm{L}$ and R. Each inner probe works as a current splitting Hall-effect sensor. In case of zero magnetic field and assuming a complete symmetry and homogeneity of the active region, the current density $J_{A}$ splits into two equal current densities $J_{A, L}$ and $J_{A, R}$ and the voltage potential $V_{A}$ between contacts 1 and 2 is zero.

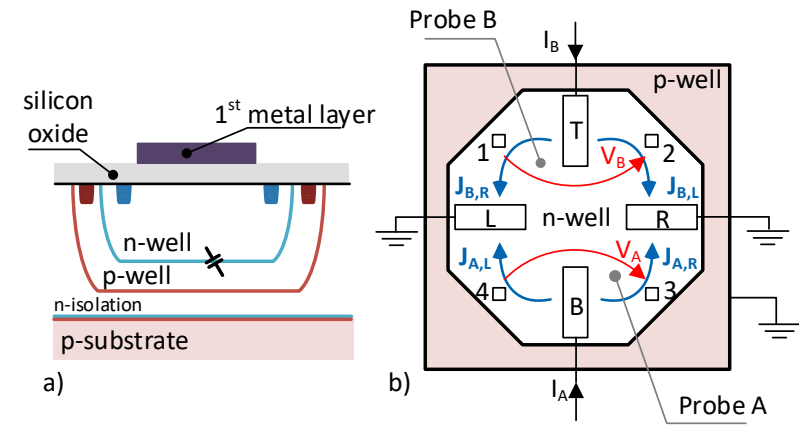

Fig. 1. (a) Vertical cross-section of the X-Hall probe showing the encapsulation of the active magnetic-sensitive region by a surrounding p-well and highlighting the presence of parasitic capacitance due to the reversed-biased junction. (b) Top view of the X-Hall probe showing its geometrical design. The figure also shows the connection of the bias contacts and the nominal current densities flowing through the probe.
The presence of a magnetic field $B_{Z}$, which is orthogonally applied to the plane identified by the active region, creates a current imbalance and the Hall voltage appears between contacts 1 and 2, so that $V_{A}=V_{H}$. Asymmetries along the horizontal axis, as well as global inhomogeneities (e.g. a resistivity gradient), gives origin to an additive offset voltage:

$$
V_{A}=V_{H}+V_{O S}^{(A)}
$$

Inner probe $\mathrm{B}$ behaves in the same identical manner but generates a Hall voltage with opposite sign, since the bias current flows in the opposite direction. Thus, voltage $V_{B}$ can be written as:

$$
V_{B}=-V_{H}+V_{O S}^{(B)},
$$

where it is reasonable to assume that the offset voltage $V_{o s}^{(B)}$, even though different in value, has the same sign of $V_{o s}^{(A)}$, given that the two inner probes share the same active region. Thus, it is very likely that the main source of offset is the same in both the inner probes.

The X-Hall architecture is completed by a crossconnection of the sense contacts along with the diagonal directions (i.e. 1 with 3 and 2 with 4 ) setting the equality

$$
V_{A}=-V_{B}
$$

Under the hypothesis of concordant offset voltages and substituting (1) and (2) into (3), then the only possible solution to (3) implies the equality

$$
V_{O S}^{(A)}=V_{O S}^{(B)}=0 \mathrm{~V}
$$

From a physical perspective, the cross short circuits add a boundary condition to the net charge distribution that forces the minimization of the offset contributions to voltages $V_{A}$ and $V_{B}$. More precisely, if the physical origin and sign of the offset are rigorously the same for both the elementary probes, then the only value of offset that satisfies both the symmetry of the probe and the boundary condition imposed by the short circuits is zero. Actually, there will always be present uncorrelated local defects or asymmetries that lead to a residual offset voltage $\Delta V_{O S}$ that adds to the voltage $V_{\text {probe }}=$ $V_{A}=-V_{B}$

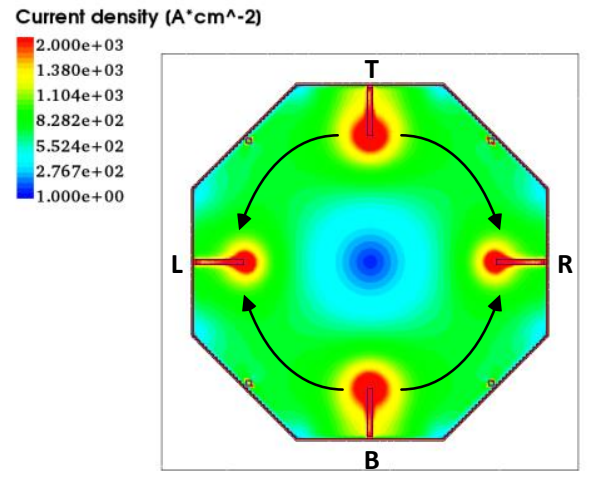

Fig. 2. TCAD simulation of the current distribution over the octagonal probe accordingly to the X-Hall biasing scheme $(500-\mu \mathrm{A}$ currents injected into B and $\mathrm{T}$ contacts with no magnetic field applied). 


\section{Bandwidth Enhancement}

Papers [5] and [7] recognize four bandwidth limits (ordered by decreasing frequency): $i$ ) a physical limit at $1 \mathrm{GHz}$ and above set by the relaxation time of the charge carriers [10]; ii) a fundamental limit set by the intrinsic capacitance created by the encapsulation of the active region in a p-type well (see Fig. 1/a); iii) a practical limit set by the capacitive load added by the electronic circuits connected to the probe, and $i v)$ a methodological limit set by the spinning-current technique [11]-[13]. This last limit is a soft one because it represents the abrupt degradation of the effectiveness of the spinning-current technique in offset reduction when it is operated at high spinning frequencies.

The passive offset reduction provided by the X-Hall architecture allows getting rid of the spinning-current technique, thus removing the methodological bandwidth limit. Moreover, the spinning-current technique is usually implemented by a great number of switches (of large sizes) and dedicated circuits that add their own capacitive load. Thus, removing the spinning-current technique has also a beneficial effect on the practical bandwidth limit by reducing the overall capacitive load. To further push the practical limit to higher frequencies, a differential-difference current feedback amplifier (DDCFA) with reduced input capacitance was designed. The differential voltage $V_{\text {probe }}$ at the output of the X-Hall probe can be directly connected to the gate of the input transistors of the DDCFA, thus only the gate capacitance, which was minimized, loads the Hall probe. Moreover, the current-feedback topology allows realizing an amplifier with adequate voltage gain and wide bandwidth, which is challenging to be implemented in smart-power technologies.

\section{STATIC CHARACTERIZATION}

\section{A. Prototype and Measurement Setup}

A first realized silicon chip implements two different prototypes (Fig. 3). The first prototype (sensor-1 in Fig. 3) consists of the octagonal Hall probe with all the bias and sense contacts accessible from the outside of the chip. This prototype was specifically designed to better estimate the offset of the X-Hall probe. The polarization of the surrounding p-type well is kept separated from the substrate potential and can be arbitrarily set at a voltage potential lower than ground. In the following tests, the p-type well is kept connected to ground. A copper metal strip on the last metal layer is used to create a path for high-value current generating the magnetic field to be measured. The second prototype (sensor-2 in Fig. 3 ) integrates in the silicon chip a bias circuit and a simple differential-difference amplifier (DDA) together with the XHall probe. The integrated generation of the bias current ensures lower noise and interferences, while the amplifier allows measuring higher output voltages, which are easier to measure. The silicon chip is encapsulated into a power small outline (PSSO) package for better thermal management.

\section{B. Residual Offset}

The setup implemented for offset measurements is shown in Fig. 4, where the first prototype was used to avoid introducing non-idealities and offsets from the integrated amplifier and to ensure better control of the bias currents. The sensor was mounted on a spring socket for statistical testing on all the realized samples. A two-channel 2602B sourcemeasurement-unit (SMU) was used to bias the Hall probe with two nominally equal currents $I_{A}=I_{B}$. The differential $V_{\text {probe }}$

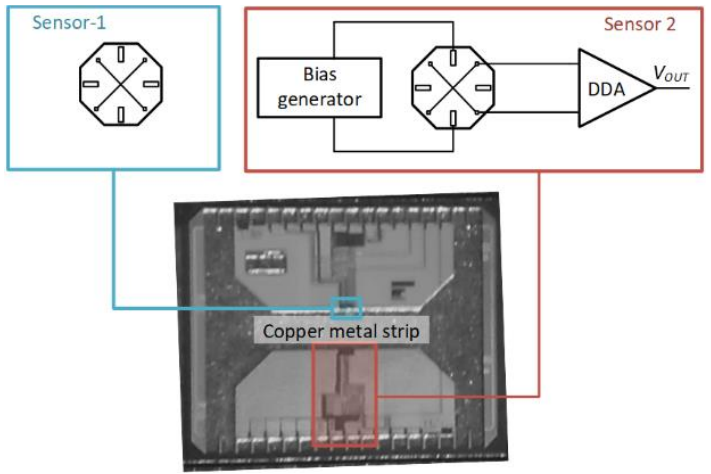

Fig. 3. First silicon chip realized in BCD8 technology from STMicroelectronics. The chip integrates two X-Hall sensor prototypes: sensor- 1 is composed of only the octagonal X-Hall probe with all the contacts accessible; sensor-2 is composed of the X-Hall probe, the generator of the bias currents and a DDA.

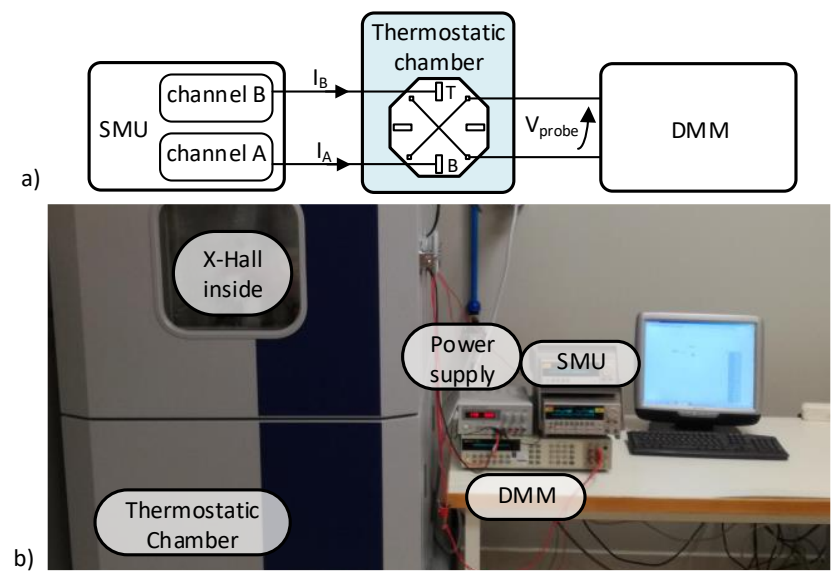

Fig. 4. (a) Setup implemented for the estimation of the offset and its time dispersion: schematic diagram and (b) photograph.

voltage at the output of the Hall probe was measured by using the $81 / 2$-digit Keysight 3458 -A digital multimeter (DMM) set at the minimum range of $100 \mathrm{mV}$, with a rated uncertainty of $0.7 \mu \mathrm{V}$. The sensor prototype was placed inside a thermostatic chamber with a temperature of $25.0(3){ }^{\circ} \mathrm{C}$. No current flowed through the copper metal strip to model the output voltage of the probe as:

$$
V_{\text {probe }}=\Delta V_{\text {oS }}+S_{I} I_{\text {bias }} B_{\text {earth }}
$$

where $B_{\text {earth }}$ is the earth magnetic field estimated according to the International Geomagnetic Reference Field (IGRF) model and $I_{\text {bias }}=I_{A}=I_{B}$. From (5), the residual offset $\Delta V_{O S}$ of the XHall probe can be estimated by directly measuring the output voltage of the probe and subtracting the estimated effect of the earth magnetic field. The measurement procedure was repeated over all the 12 available samples with $I_{\text {bias }}=1 \mathrm{~mA}$. The estimated mean residual offset is $-0.513(13) \mathrm{mV}$ with standard deviation of $1 \mathrm{mV}$ over the sample population. This value corresponds to a mean input-referred offset of -2.05(5) $\mathrm{mT}$. The number of available samples is not sufficient to provide a comprehensive statistical analysis of the residual offset; nonetheless, a preliminary study on the effectiveness of the X-Hall probe can still be undertaken.

Compared to the state of the art [3], [4], [11], [14], the XHall sensor has a residual offset that is considerably higher, but this is related also to technological and implementation aspects. To correctly assess the effectiveness of the X-Hall 
sensor, it must be compared to a Hall-effect sensor realized in the same silicon technology. In [5], a spinning-currentoperated Hall-effect sensor implemented in the same BCD technology was presented and characterized. It showed a mean input-referred offset that ranged between $350 \mu \mathrm{T}$ (when operated at low frequencies) and $17 \mathrm{mT}$ (when operated at frequencies higher than $1 \mathrm{MHz}$ ). The X-Hall sensor prototype shows higher offset if compared to [5] when the latter is operated at low frequencies but features much lower offset if compared to [5] when operated at high frequencies. In summary, the X-Hall architecture offers an intermediateeffective but strongly frequency-independent offset reduction, allowing for much wider acquisition bandwidth while preserving basically the same offset rejection capability. Thus, the X-Hall sensor is an interesting solution when the final application requires high operating frequencies with a still acceptably low offset.

Short- and long-term stability of the offset is even more important than the estimated offset value since one-point correction is easily implementable. To assess the long-term stability of the residual offset, a single chip prototype was placed inside the thermostatic chamber, with temperature set to $25.0(3){ }^{\circ} \mathrm{C}$ and constantly biased and monitored for two days, with a sampling time of 10 minutes. The recorded time dispersion of the offset voltage is reported in Fig. 5, showing a stable residual voltage with a maximum variation of 20(4) $\mu \mathrm{V}$, which corresponds to $80(16) \mu \mathrm{T}$. This is a promising result, although a longer recording is needed to assure the low time dispersion of the residual offset. Evaluation of the shortterm stability is also required to prove the general stability of the offset in the X-Hall sensor.

\section{Static Characteristic}

The static characteristic was evaluated on the prototype described in Section III-A as sensor-2. A Keysight E3633A power supply was connected to the copper metal strip through a $1-\Omega 50-\mathrm{W}$ resistor to generate an input current which is then measured by using a $6 \frac{1}{2}$-digit Keysight 34401 DMM. The current flowed through the copper metal strip placed above the Hall sensor and generated a magnetic field with a transduction factor of $2 \mathrm{mT} / \mathrm{A}$ (data from physical simulations). The power supply was programmed to generate pulsed currents $\left(T_{O N}=\right.$ $600 \mathrm{~ms}$, duty cycle $=1 \%$ ) to not cause excessive heating of the sensor (overheating controlled to be under $5{ }^{\circ} \mathrm{C}$ at the package surface), since the realized prototype is not provided of a temperature compensation circuits (which is standard and it is not interesting for the scope of this manuscript). The

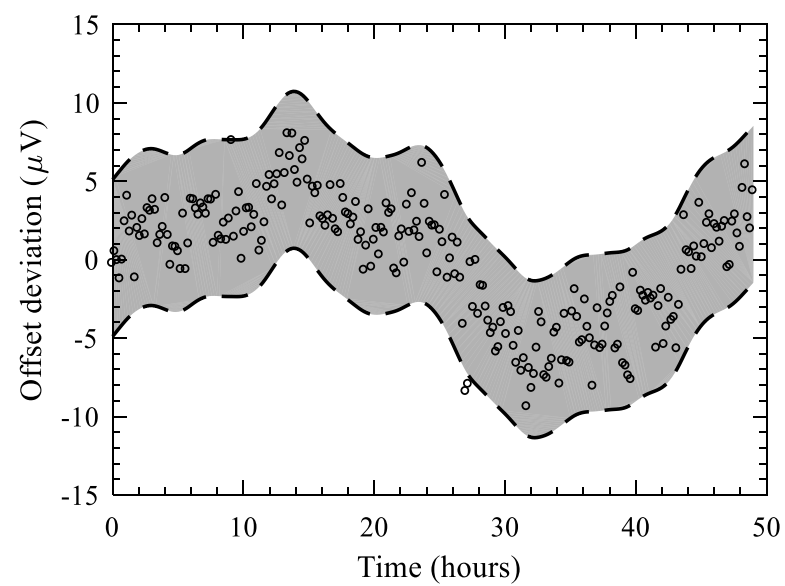

Fig. 5. Time dispersion of the residual offset over a 2-day acquisition. The shaded region defines the uncertainty band of the measurement. voltage $V_{\text {OUT }}$ at the output of the amplifier was recorded by the Keysight 3458-A DMM.

The static characteristic over the $\pm 10 \mathrm{~A}( \pm 20 \mathrm{mT})$ input range was measured for two bias currents, $I_{\text {bias }}=0.9 \mathrm{~mA}$ and $I_{\text {bias }}=0.5 \mathrm{~mA}$, and is shown in Fig. 6 . The total estimated sensitivity $G$ is $36 \mathrm{mV} / \mathrm{A}$ and $23 \mathrm{mV} / \mathrm{A}$, respectively. These sensitivity values take into account the current-to-magnetic field transduction $G_{I B}$, the sensitivity of the Hall probe $G_{H}$ and the gain of the electronic amplifier $G_{E L E}$ :

$$
G=G_{I B} \cdot G_{H} \cdot G_{E L E} \cdot
$$

By assuming $G_{I B}=2 \mathrm{mT} / \mathrm{A}$ and $G_{E L E}=100 \mathrm{~V} / \mathrm{V}$, then the sensitivity of the Hall probe is $180 \mathrm{mV} / \mathrm{T}$ for $I_{\text {bias }}=0.9 \mathrm{~mA}$ and $115 \mathrm{mV} / \mathrm{T}$ for $I_{\text {bias }}=0.5 \mathrm{~mA}$, corresponding to a currentrelated sensitivity

$$
S_{I}=\frac{G_{H}}{I_{\text {bias }}}
$$

ranging between $200 \mathrm{~V} / \mathrm{AT}$ and $230 \mathrm{~V} / \mathrm{AT}$, which is in agreement with the state of the art [5], [15], [16].

\section{DYNAMIC CHARACTERIZATION}

\section{A. Prototype and Measurement Setup}

To correctly assess the dynamic performance of the X-Hall architecture, a third prototype (sensor-3) was realized on a different silicon chip. This prototype has the same architecture of sensor-2 but replaces the standard DDA with a broadband current-feedback differential-difference amplifier (CFDDA). Current-feedback amplifiers allow to achieve a much higher bandwidth but suffer from higher offset and noise. The CFDDA used in this prototype has a closed-loop bandwidth of $65 \mathrm{MHz}$, as from simulation results. The X-Hall sensor chip was mounted on a dedicated board with reduced dimensions and improved signal integrity solutions (e.g., a coplanar strip for the delivery of the high-frequency input current, the use of capacitor arrays on the power supplies, the minimization of inductive parasitics at board level, etc.). A photograph of the realized test board, together with the measurement setup, is reported in Fig. 7.

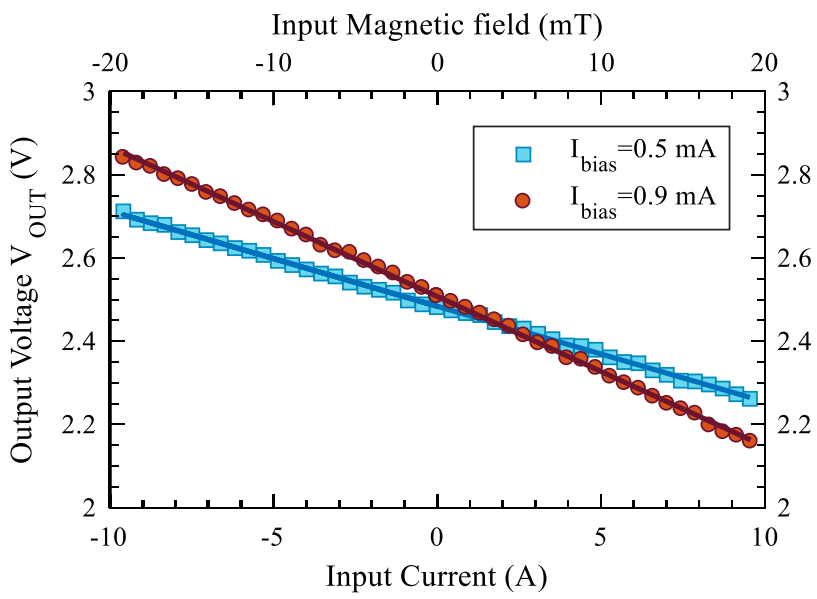

Fig. 6. Static characteristic. 


\section{B. Transfer Function}

The transfer function (TF) was estimated by applying a 200-mA-amplitude sinewave at known frequencies to a $50-\Omega$ resistor connected in series to the copper metal strip on the top of the metal stack inside the silicon chip. The sinewave is generated by the Keysight $81150 \mathrm{~A}$ arbitrary function generator (AFG) and monitored by the $100-\mathrm{MHz}$ bandwidth N2783A current probe connected to the Keysight DSO9254A oscilloscope. The output of the X-Hall sensor (i.e., the voltage $V_{\text {OUT }}$ at amplifier output) is synchronously acquired by the same oscilloscope sampling at a mean rate of $1 \mathrm{GSa} / \mathrm{s}$. Coherent averaging over multiple acquisitions is performed to improve the final resolution of the measurement. Finally, TF estimation was performed in MATLAB. The measurement setup excited the sensor with approximately 10 frequency points per decade from $1 \mathrm{kHz}$ to $20 \mathrm{MHz}$.

Fig. 8 shows the estimated TF for different bias currents. The absolute value of the low-frequency gain is slightly different from that reported in Section III-C since the two measured values belong to two different prototypes. Increasing the bias current boosts the low-frequency gain, as also expected from static characterization. At higher frequencies, the TF rises by about $20 \mathrm{~dB} /$ decade due to the presence of additive perturbative effects, which are not related to the X-Hall architecture but depend on the implementation of the prototype (see below for brief discussion). To prove this statement, the same measurement procedure was repeated with no bias current flowing through the Hall probe, thus nulling the magnetic field and the Hall voltage. Moreover, all the bias contacts of the probe were directly connected to the same voltage provided by an external voltage generator in order to null the offset voltage, too. The result of this measurement is shown by the black line in Fig. 8, which still presents the parasitic behavior at frequencies higher than 200 $\mathrm{kHz}$. As a result, the output of the realized X-Hall prototype can be written as:

$$
V_{\text {OUT }}=G \cdot I_{I N}+\Delta V
$$

where $\Delta V$ represents the superposition of the dynamic perturbations.

The physical origins of these parasitic effects are still under investigation. They could be ascribed to inductive coupling between the copper metal strip (in which the input current flows) and the input nodes of the CFDDA, or to a capacitive coupling to the internal power supply rails that reverberates across the CFDDA, which has a poor PSRR at high frequencies, or even a combination of both effects. Regardless of the physical origin of the perturbative effect, it is possible to compensate for it by a vector differential procedure, leading to the following TF of the X-Hall sensor as:

$$
G(f)=\frac{V_{\text {OUT }}(f)-\Delta V(f)}{I_{I N}(f)}
$$

Fig. 9 reports the result of the compensation procedure modelled by (9). From this experiment, it is possible to appreciate an acquisition bandwidth, defined as a 3-dB deviation from the flatness, of about $4 \mathrm{MHz}$. Due to the differential nature of the compensation technique described by (9), the relative uncertainty of the estimated TF for frequency

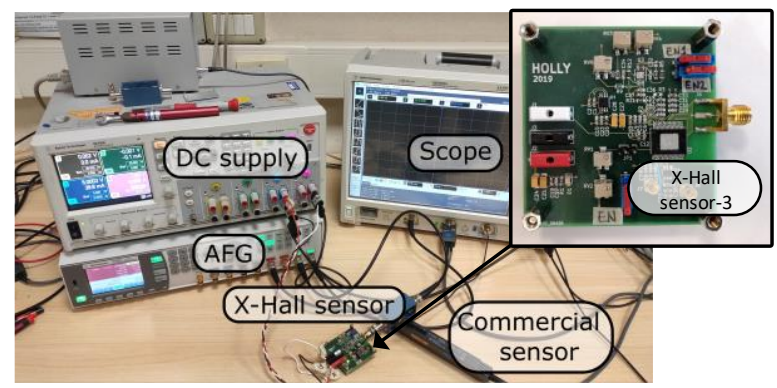

Fig. 7. Measurement setup and test board used for the dynamic characterization of the X-Hall architecture by using the sensor-3 prototype.

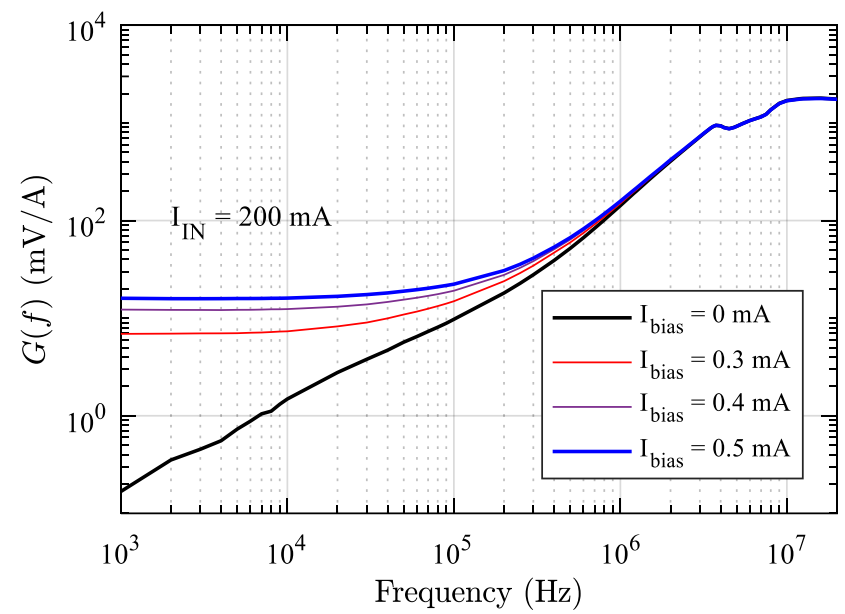

Fig. 8. TF of X-Hall sensor-3 prototype estimated for different bias currents.

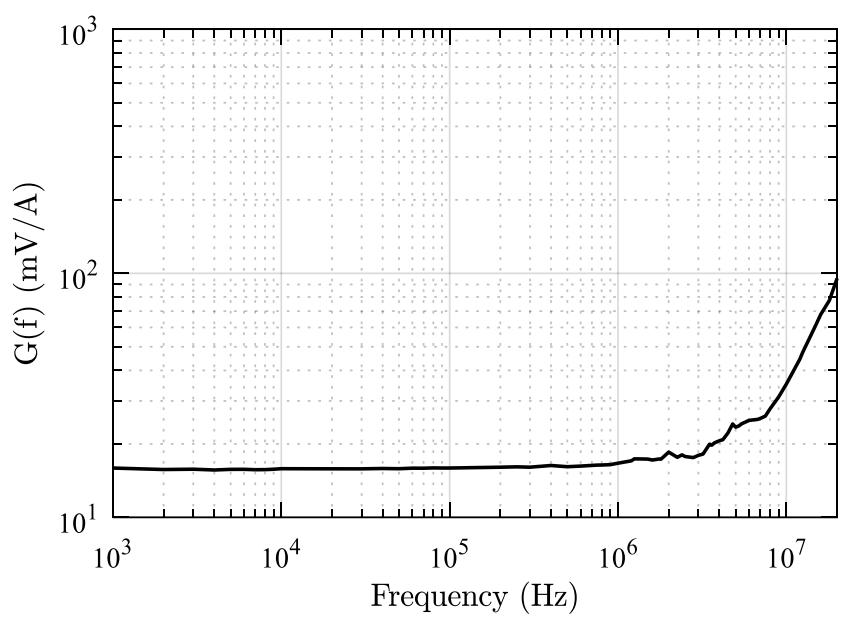

Fig. 9. Estimated TF of the X-Hall sensor after de-embedding of the dynamic perturbation.

higher than $10 \mathrm{MHz}$ becomes so high to make the results unreliable. Nonetheless, to the best knowledge of the authors, 4-MHz bandwidth is the broadest bandwidth ever achieved by a purely Hall-effect based sensor, although still far from the result achieved in simulation that is very close to the practical limit. Fig. 10 reports the simulated dynamic response of the $\mathrm{X}$-Hall output voltage to a $50-\mathrm{mT}$ step of the input magnetic field at $t=0 \mathrm{~s}$. The simulation took into account a bias current of $500 \mu \mathrm{A}$ and an equivalent input capacitance of the electronic amplifier of $500 \mathrm{fF}$. The simulation was realized by using Sentaurus TCAD with the model described in [9], and [17]. According to the simulation result, the X-Hall sensor could ideally achieve a bandwidth higher than $200 \mathrm{MHz}$ if $i$ ) 


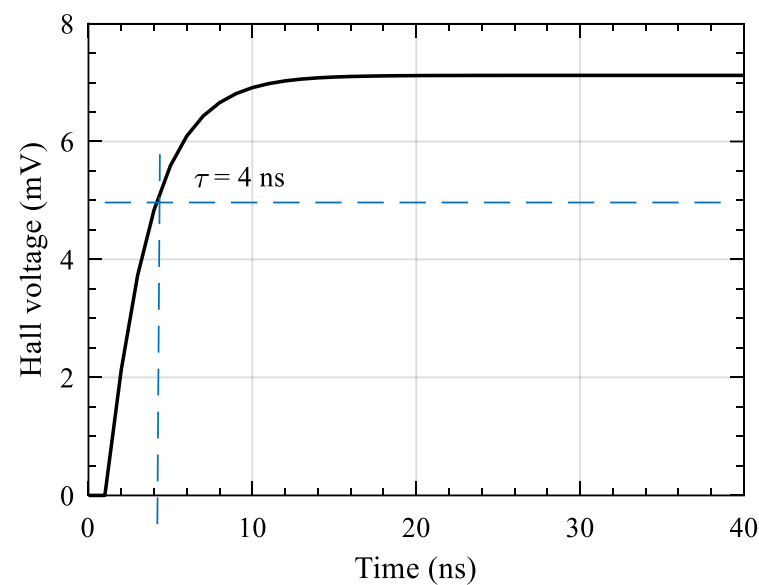

Fig. 10. TCAD simulation result of the theoretical response of the X-Hall sensor to a 50-mT step of magnetic field. The X-Hall probe is biased by a $500 \mu \mathrm{A}$ and a $500 \mathrm{fF}$ capacitor is connected to the output to simulate the loading effect of the amplifier. The simulation shows a practical bandwidth limit higher than $200 \mathrm{MHz}$

the dynamic perturbations are made negligible by improving the prototype implementation, ii) the capacitance of the amplifier is minimized to less than $500 \mathrm{fF}$, and iii) the bandwidth of the amplifier is higher than $200 \mathrm{MHz}$.

\section{CONCLUSION}

This paper experimentally investigated the potentiality of the X-Hall architecture for integrated, broadband measurement of magnetic field or current. The X-Hall sensor overcomes the bandwidth limit of state-of-the-art Hall sensors by replacing the spinning-current technique with a DC-biasbased, passive offset compensation, which is less effective but frequency independent. The elimination of the spinningcurrent technique removes the methodological bandwidth limit and push the practical limit close to the fundamental limit. The X-Hall sensor could theoretically achieve a bandwidth as high as $200 \mathrm{MHz}$, as demonstrated by means of TCAD simulation. However, measurements on the X-Hall prototypes identified the presence of a additive dynamic perturbations that set a new bandwidth limitation. These perturbations are related to the practical implementation of the sensor and may be ascribed to non-ideal magnetic/electric isolation of the sensor from the origin of the magnetic field. Based on a vector differential model, it was possible to compensate for the dynamic perturbation and experimentally achieve a sensor bandwidth of $4 \mathrm{MHz}$, which is the broadest bandwidth ever achieved by a purely Hall-effect based sensor. The X-Hall sensor shows higher offset (approximately 6-fold increase) than low-frequency-operated spun Hall sensors but lower offset (approximately a factor 9) with respect to highfrequency-operated spun Hall sensors. Moreover, the X-Hall sensor also promises low time dispersion of the residual offset, which is of more importance than the absolute value of the offset itself.

\section{ACKNOWLEDGMENT}

The authors would like to thank M. Biondi of the University of Bologna for the design of the DDCFA and for the discussions on the $\mathrm{X}-\mathrm{Hall}$ architecture.

This work was partially supported by the ECSEL Joint Undertaking (JU) through EU H2020 program and Italy, Germany, The Netherlands, Spain, and Slovakia under Grant Agreement no. 737434.

\section{REFERENCES}

[1] Y. H. Lee et al., "Power-tracking embedded buck-boost converter with fast dynamic voltage scaling for the SoC system," IEEE Trans. Power Electron., vol. 27, no. 3, pp. 1271-1282, 2012.

[2] W. Jin, A. T. L. Lee, S.-C. Tan, and S. Y. Hui, "A Gallium Nitride (GaN)-Based Single-Inductor Multiple-Output (SIMO) Inverter With Multi-Frequency AC Outputs," IEEE Trans. Power Electron., vol. 34, no. 11, pp. 10856-10873, Jan. 2019.

[3] J. Jiang and K. A. A. Makinwa, "Multipath Wide-Bandwidth CMOS Magnetic Sensors," IEEE J. Solid-State Circuits, vol. 52, no. 1, pp. 198-209, Jan. 2017

[4] S. Huber, W. Leten, M. Ackermann, C. Schott, and O. Paul, “A Fully Integrated Analog Compensation for the Piezo-Hall Effect in a CMOS Single-Chip Hall Sensor Microsystem," IEEE Sens. J., vol. 15, no. 5, pp. 2924-2933, May 2015.

[5] M. Crescentini, M. Marchesi, A. Romani, M. Tartagni, and P. A. Traverso, "A Broadband, On-Chip Sensor Based on Hall Effect for Current Measurements in Smart Power Circuits," IEEE Trans. Instrum. Meas., vol. 67, no. 6, pp. 1470-1485, 2018.

[6] M. Oszwaldowski and S. El-Ahmar, "Double Hall sensor structure reducing voltage offset," Rev. Sci. Instrum., vol. 88, no. 7, p. 075005 , Jul. 2017.

[7] M. Crescentini, M. Marchesi, A. Romani, M. Tartagni, and P. A. Traverso, "Bandwidth Limits in Hall Effect-based Current Sensors," ACTA IMEKO, vol. 6, no. 4, p. 17, Dec. 2017.

[8] M. Crescentini, M. Biondi, M. Marchesi, A. Romani, M. Tartagni, and P. A. Traverso, "Bandwidth enhancement in Hall probe by X-Hall DC biasing," J. Phys. Conf. Ser., vol. 1065, no. 5, p. 052031, Aug. 2018.

[9] M. Crescentini, M. Biondi, A. Romani, M. Tartagni, and E. Sangiorgi, "Optimum Design Rules for CMOS Hall Sensors," Sensors, vol. 17, no. 4, p. 765, Apr. 2017.

[10] R. S. Popovic, Hall Effect Devices, 2nd ed. Boca Raton (FL): CRC Press, 2004

[11] V. Mosser, N. Matringe, and Y. Haddab, "A Spinning Current Circuit for Hall Measurements Down to the Nanotesla Range," IEEE Trans. Instrum. Meas., vol. 66, no. 4, pp. 637-650, Apr. 2017.

[12] U. Ausserlechner, "Limits of offset cancellation by the principle of spinning current hall probe," in Proceedings of IEEE Sensors, 2004., 2004, pp. 1117-1120.

[13] P. J. A. Munter, "A low-offset spinning-current hall plate," Sensors Actuators A Phys., vol. 22, no. 1-3, pp. 743-746, Jun. 1990.

[14] Y. Li, M. Motz, and L. Raghavan, "A Fast T\&amp;H Overcurrent Detector for a Spinning Hall Current Sensor With Ping-Pong and Chopping Techniques," IEEE J. Solid-State Circuits, vol. 54, no. 7, pp. 1852-1861, Jul. 2019.

[15] J. Jiang and K. Makinwa, "A hybrid multipath CMOS magnetic sensor with $210 \mu$ Trms resolution and $3 \mathrm{MHz}$ bandwidth for contactless current sensing," in IEEE Int. Solid-State Circuits Conf. (ISSCC) Dig. Tech. Papers, 2016, pp. 204-205.

[16] T. Funk and B. Wicht, "A Fully Integrated DC to $75 \mathrm{MHz}$ Current Sensing Circuit with On-Chip Rogowski Coil,” pp. 6-9, 2018.

[17] M. Crescentini, A. Romani, and E. Sangiorgi, "Physical simulations of response time in Hall sensor devices," in 2014 15th International Conference on Ultimate Integration on Silicon (ULIS), 2014, pp. 8992. 